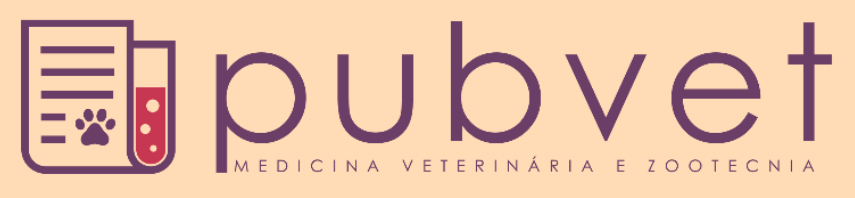

https://doi.org/10.31533/pubvet.v12n9a170.1-9

\title{
Bloqueio de nervos femoral e isquiático em cirurgias ortopédicas de pequenos animais
}

\author{
Emanuelle Bortolotto Degregori ${ }^{* \bullet}$, Nathalia Franco ${ }^{2}{ }^{\ominus}$, Matheus da Rosa Pippi ${ }^{3}{ }^{\bullet}$, \\ Luciana Gonçalves Teixeira ${ }^{4}{ }^{\bullet}$, Emerson Antonio Contesini $^{5}{ }^{\circ}$
}

${ }^{1}$ Mestranda no Programa de Pós-Graduação em Ciências Veterinária, Universidade Federal do Rio Grande do Sul. Porto Alegre-RS, Brasil. ${ }^{2}$ Mestranda no Programa de Pós-Graduação em Ciências Veterinária, Universidade Federal do Rio Grande do Sul. Porto Alegre-RS, Brasil. ${ }^{3}$ Doutorando no Programa de Pós-Graduação em Ciências Veterinárias, Universidade Federal do Rio Grande do Sul. Porto Alegre-RS, Brasil. ${ }^{4}$ Doutorando no Programa de Pós-Graduação em Ciências Veterinárias, Universidade Federal do Rio Grande do Sul. Porto Alegre-RS, Brasil. ${ }^{5}$ Professor da Universidade Federal do Rio Grande do Sul, Faculdade de Veterinária. Porto Alegre-RS, Brasil. E-mail: emerson.contesini@ufrgs.br *Autor para correspondência. E-mail: emanuelle.bortolotto@gmail.com

RESUMO. A anestesia loco-regional em nervo femoral e isquiático vem sendo amplamente utilizada em medicina veterinária, pois é conhecida por reduzir a necessidade de anestésicos gerais e medicamentos analgésicos no pós-operatório, diminuindo os seus efeitos colaterais associados. Com essa revisão, objetiva-se maior entendimento sobre bloqueio dos nervos ciático e femoral, abordando suas características principais, quando utilizados em procedimentos cirúrgicos dos membros posteriores em pequenos animais. Ao comparar com anestesia peridural, maiores estudos são necessários, porém diversos trabalhos já foram realizados e publicados, tanto em humanos quanto em animais, e relataram o sucesso na redução do limiar da dor em procedimentos cirúrgicos ortopédicos.

Palavras chave: analgesia, anestesia, dor, pós-operatório, cirurgias ortopédicas

\section{Femoral and sciatic nerve block in small animal orthopedic surgeries}

\begin{abstract}
Loco-regional anesthesia in the femoral and sciatic nerve has been widely used in veterinary medicine, since it is known to reduce the need for general anesthetics and analgesic drugs in the postoperative period, reducing their associated side effects. With this review, a greater understanding of sciatic and femoral nerve block is sought, addressing its main characteristics, when used in surgical procedures of the hind limbs in small animals. When comparing with epidural anesthesia, more studies are needed, but several studies have already been performed and published, both in humans and in animals, and have reported success in reducing the pain threshold in orthopedic surgical procedures.
\end{abstract}

Keywords: analgesia, anesthesia, pain, postoperative, orthopedic surgeries

\section{Bloqueo de nervios femoral e isquiático en cirugías ortopédicas de pequeños animales}

RESUMEN. La anestesia loco-regional en nervio femoral e isquiático viene siendo ampliamente utilizada en medicina veterinaria, pues es conocida por reducir la necesidad de anestésicos generales y medicamentos analgésicos en el postoperatorio, disminuyendo sus efectos colaterales asociados. Con esta revisión, se objetiva mayor entendimiento sobre bloqueo de los nervios ciático y femoral, abordando sus características principales, cuando se utilizan en procedimientos quirúrgicos de los miembros posteriores en pequeños animales. Al comparar con anestesia peridural, mayores estudios son necesarios, pero diversos trabajos ya fueron realizados y publicados, tanto en humanos como en animales, 
y relataron el éxito en la reducción del umbral del dolor en procedimientos quirúrgicos ortopédicos.

Palabras clave: analgesia, anestesia, dolor, postoperatorio, cirugías ortopédicas

\section{Introdução}

Atualmente, o tratamento da dor pósoperatória vem ganhando ênfase, uma vez que a injúria cirúrgica é um potente estimulante de respostas neuronais, segmentares e suprasegmentares, causadores de alterações fisiológicas e complicações pós-operatórias, levando a graves repercussões em diferentes órgãos e sistemas (Gaynor, 1999).

A anestesia loco-regional é um método utilizado mediante a aplicação de um agente anestésico próximo a um nervo ou grupo de nervos de determinada região (Gebeyehua, 2014), onde ocorrerá dessensibilização da área por ele inervada. Seu uso na medicina veterinária tem ganhado enfoque nos últimos anos, sendo visto como um componente essencial de uma analgesia balanceada (Schroeder, 2013).

Por assumir preponderância na prática anestésica, permite maior eficácia analgésica no pós-operatório, reduzindo as taxas de morbidade de mortalidade, além de estar associada a baixos custos (Silva et al., 2011). Quando comparado ao uso de fármacos opioides sistêmicos, os bloqueios loco-regionais são eficazes na analgesia de pacientes submetidos a cirurgias ortopédicas (Vettorato et al., 2012).

O bloqueio dos nervos femoral e isquiático são referidos como alternativas à anestesia epidural (Campoy et al., 2008; Portela et al., 2010; Campoy et al., 2012a; Caniglia et al., 2012). Mesmo sendo considerado consagrado em medicina veterinária, o bloqueio epidural apresenta algumas complicações e efeitos indesejados, o que tornou necessária a busca por meios alternativos para anestesia dos membros pélvicos (Portela, 2013; Gurney \& Leece, 2014).

Esta revisão tem como objetivo, obter maior entendimento sobre bloqueio dos nervos ciático e femoral, abordando suas características principais, quando utilizados em procedimentos cirúrgicos dos membros posteriores em pequenos animais.

\section{Material e Métodos}

Para esta pesquisa foram inseridos no portal periódicos CAPES, Pubmed e Google Scholar, os termos "loco-regional anesthesia", "dog", "cat", "analgesia", "orthopedic surgery" "pain" "pelvic limbs", "femoral nerve", "sciatic nerve". Ainda, estes termos foram procurados em livros relacionados ao tema em questão. Após a leitura, as informações foram sistematizadas e apresentadas em forma de revisão de literatura.

\section{Desenvolvimento}

\section{Dor}

A dor é descrita em termos de estímulos nocivos, revelando-se danosa ou potencialmente prejudicial aos tecidos (Muir, 2009). Trata-se de um meio de proteção do organismo, ativado quando é chegada a hora de "parar" com o estímulo que a gerou (Ferreira \& Obertz, 2013).

Pode ser classificada em aguda, quando advém de lesões traumáticas, procedimentos cirúrgicos ou infecção, tendo surgimento repentino e com duração limitada (Treede et al., 2008). Esta, por sua vez, quando não tratada no pós-operatório, ou tratada de maneira ineficaz, acarreta em dor patológica, influenciando de maneira negativa a recuperação do paciente (Anil et al., 2002; Hoelzer et al., 2005; Bufalari et al., 2007). Já a dor crônica, é considerada um estado doloroso constante, observado por longo período de tempo, ou que retorna após lesão ou trauma tecidual (Treede et al., 2008). O estabelecimento de terapia analgésica apropriada, reduz os efeitos colaterais provocados pela dor, sejam eles metabólicos, imunológicos ou endócrinos. A seu favor, a terapia analgésica reduz a morbidade e mortalidade pós-operatória $(\underline{\mathrm{Xu} \& \text { Brennan, }}$ 2011).

Existem diversos meios de reduzir a intensidade da dor pós-operatória de pacientes submetidos a cirurgias ortopédicas, tendo como exemplo, a anestesia loco-regional. Esta tem sido amplamente empregada com o intuito de reduzir a quantidade requerida de anestésicos gerais, reduzindo seus riscos associados (Gurney \& Leece, 2014). Os anestésicos locais atuam nos canais de sódio, de forma a impedir a entrada rápida deste íon na célula. $O$ bloqueio dos canais de sódio possibilita a estabilização da membrana, interditando a deflagração e condução do estímulo elétrico (Becker \& Reed, 2012). A administração desses fármacos pode ser executada por distintas 
vias, como tópica, infiltrativa, perineural, intravenosa e espinhal, sendo que, o êxito da técnica está correlacionado à precisão do local de deposição do anestésico local (Mahler \& Adogwa, 2008).

O reconhecimento da dor, em medicina veterinária, é desafiador e subjetivo à análise do observador. Por tratar-se de sensação, a dor é particular de cada indivíduo, e é nesse momento onde há maior dificuldade de identificação no paciente veterinário (Flôr et al., 2011). Segundo Mich \& Hellyer (2009), a experiência do avaliador é muito importante, para que os parâmetros não sejam super ou subestimados. Além disso, para reduzir a interferência, seria necessário período de adaptação dos animais com o avaliador.

\section{Anestesia loco-regional}

A aplicação de anestésicos locais e analgésicos por via epidural tem sido amplamente empregada em associação, ou como método alternativo da anestesia geral em procedimentos ortopédicos em membros pélvicos de pequenos animais (Caniglia et al., 2012). O bloqueio dos nervos femoral e ciático são retratados como opção para promover anestesia e analgesia. São aconselháveis naqueles pacientes em que a anestesia peridural é contraindicada ou quando o bloqueio bilateral não é desejado (Mahler \& Adogwa, 2008; Campoy et al., 2010; Portela et al., 2013).

Quando contraposto à anestesia geral e terapia analgésica sistêmica, a anestesia loco-regional proporciona inúmeros benefícios, como efeitos anti-inflamatórios, superior recuperação funcional (Mahler \& Adogwa, 2008), estabilidade cardiorrespiratória elevada, menor permanência hospitalar (Chery et al., 2014), retorno rápido à função gastrointestinal, menor depressão imunológica (Kurosawa \& Kato, 2008), além de influenciar positivamente na função cognitiva pós-operatória (Davis et al., 2014) e reduzir as chances de recidivas neoplásicas (Conrick-Martin \& Buggy, 2013). Por ser considerada mais segura, quando comparada a anestesia geral, pode ser usada em animais portadores de cardiopatias, hepatopatias, nefropatias, alterações endócrinas, neurológicas e em pacientes idosos, que muitas vezes apresentam comorbidades (Kahvegian, 2010)

Estes procedimentos são bem quistos na rotina, pois possuem grande aplicabilidade, fácil execução e gerar pouco desconforto para os pacientes. Porém, para obter uma técnica bemsucedida, faz-se necessário o conhecimento anatômico regional, com o intuito de estabelecer pontos de referência que sejam fidedignos, bem como os locais de punção, direção e profundidade da agulha e quais as estruturas importantes devem ser evitadas (Gurney \& Leece, 2014; Gebeyehua, 2014).

\section{Anatomia dos nervos femoral e ciático}

Os plexos sacral e lombar são os principais responsáveis pela inervação do membro pélvico. O bloqueio do membro pélvico pode ser realizado com a administração de anestésicos nos seguimentos do plexo lombossacro: nervos femoral, ciático, femoral lateral cutâneo, obturador e femoral caudal cutâneo (Portela et al., 2010; Portela, 2013). Os nervos femoral e ciático são os principais ramos do plexo lombossacro, e o bloqueio dos mesmos resulta em anestesia do membro pélvico quase que em sua totalidade (Campoy et al., 2008; Mahler \& Adogwa, 2008; Portela, 2013)

O nervo femoral é o principal dos nervos do plexo lombar, ele se direciona caudalmente, emerge no triângulo femoral e posteriormente emite um ramo cutâneo, sendo conhecido por nervo safeno. Participa da inervação da região dorsomedial da coxa, medial do tarso, articulação femorotibiopatelar, cápsula articular e estruturas internas, e também fornece inervação motora dos músculos ílio psoas, sartório e quadríceps. Já o nervo ciático tem origem dos ramos ventrais e sacrais do tronco lombossacral. Em relação a sua localização, logo após deixar a cavidade pélvica através do forame ciático maior, encontra-se medialmente com os músculos glúteos, gastrocnêmico, e obturador interno. Segue caudalmente até o trocânter maior do fêmur e se direciona distalmente entre o trocânter e tuberosidade isquiática. Dá sequência distalmente como nervo fibular comum e nervo tibial (Portela, 2013).

Para a realização da anestesia regional do membro pélvico, deve-se administrar um anestésico local próximo aos principais nervos, femoral e ciático. Para este fim, lança-se mão do uso de eletroestimulação e ultrassom (US). Estes foram julgados quanto à possibilidade de aumentar a taxa de sucesso nos bloqueios nervosos (Campoy et al., 2010; Tayari et al., 2017).

Técnicas de anestesia regional periférica do membro posterior

Com a criação e aplicação de aparelhos que colaborassem na execussão da anestesia regional, 
como estimulador de nervos periféricos (ENP) e ultrassom (US), as técnicas de anestesia locoregional foram aperfeiçoadas, garantindo boqueio total da condução nervosa, além de anestesia segura e de qualidade. A ideia de usar aparelhos de neurolocalização é determinar a localização precisa dos nervos, ou de um grupo de nervos, possibilitando a administração correta do anestésico (Campoy, 2006).

$O$ uso de ENP proporciona melhora na qualidade dos bloqueios periféricos, reduzindo taxa de insucesso, bem como a dose do fármaco (Rodriguez et al., 2004; Mahler \& Adogwa, 2008). O principio da estimulação elétrica com ENP, se dá quando impulsos elétricos atingem o nervo e são transmitidos ao longo das fibras nervosas. A intensidade do estimulo é equivalente a distância da extremidade da agulha e o nervo, desta forma, quanto mais próximo do nervo, menor será a intensidade necessária (Marhofer \& Chan, 2007).

A utilização do US tem sido amplamente estudada há mais de 20 anos, e está se tornando mais difundida nas várias técnicas de bloqueios de nervos periféricos em pequenos animais (Mogicato et al., 2015; Tayari et al., 2017). Ele proporciona diversos benefícios sobre as abordagens, tanto às cegas, quanto estimuladores nervosos. Possibilita a visualização do procedimento em tempo real, permitindo reconhecer o nervo alvo e manipular a agulha em relação ao mesmo, além de conseguir visualizar outras estruturas anatômicas importantes (Campoy et al., 2010; Costa-Farré et al., 2011; Graff et al., 2015; Tayari et al., 2017). Além disso, permite observar a disseminação do anestésico local, a qual poderá ser depositada com maior acurácia, sem afetar a eficácia do bloqueio. Tudo isso favorece a redução da necessidade de diversas passagens da agulha, reduzindo o risco de perfuração vascular e tempo (Campoy et al., 2010; Graff et al., 2015; Tayari et al., 2017).

Recentemente, estudos experimentais têm identificado pontos de referência e acessos apropriados para o bloqueio dos nervos femoral e ciático (Campoy et al., 2008; Mahler \& Adogwa, 2008; Gurney \& Leece, 2014). Para realizar o bloqueio do nervo femoral pode-se lançar mão do acesso inguinal, na região do triângulo femoral (Mahler \& Adogwa, 2008; Portela, 2013), onde se encontra cranial a artéria femoral, a qual é usada como ponto de referência (Evans \& De Lahunta, 2010). Outra alternativa é o acesso pré-ilíaco, quando este se encontra no compartimento psoas.
Um estudo realizado por Tayari et al. (2017), demonstrou que este bloqueio realizado com auxílio do ultrassom em cães, submetidos a osteotomia de nivelamento platô tibial (TPLO) proporcionou analgesia, reduzindo a necessidade de opioides sistêmicos no pós-operatório por nove horas. A vantagem desta abordagem, segundo Portela et al. (2013), é que não há necessidade de trocar o paciente de decúbito.

Já o nervo ciático, pode ser localizado entre o trocânter maior e a tuberosidade isquiática (Campoy et al., 2008; Mahler \& Adogwa, 2008; Portela, 2013), ou então pelo acesso parassacral, onde pode ser bloqueado próximo a suas origens (L7-S2). Assim, traça-se uma linha entre a crista ilíaca e a tuberosidade isquiática, injetando o anestésico na junção do terço medial e cranial (Portela et al., 2010). Mahler \& Adogwa (2008) propuseram o acesso através do músculo glúteo, porém, este apresenta maior dificuldade de execução. Com auxílio do ultrassom, o acesso lateral é descrito sendo de fácil realização (Campoy et al., 2008; Portela et al., 2013).

A maior dificuldade relatada por Nielsen et al., (2005), foi a realização de bloqueios em animais com sobrepeso, principalmente naqueles com maior deposição de gordura na região lombar, pois dificulta a localização dos pontos de referência, fator determinante para o êxito da técnica.

\section{Bloqueio de nervos femoral e ciático em cirurgias de membros posteriores}

Cirurgias ortopédicas são procedimentos vistos como promotores de dor, de intensidade moderada a severa, sendo que a analgesia peri e pósoperatória tem sido considerada desafiadora em animais (Johnson, 2013).

Tendo por objetivo aumentar a duração da anestesia regional, controlando a dor pósoperatória, existem muitos fármacos que são descritos como adjuvantes. Uma diversidade de fármacos já foi testada, como opioides, dissociativos, agonistas $\alpha 2$-adrenérgicos (Otero et al., 2011; Marhofer et al., 2013). Um estudo realizado por Esmaoglu et al. (2010), em humanos, concluiu que a dexmedetomidina reduziu a latência e estendeu a ação motora e sensitiva, quando associada à levobupivacaína. Pesquisas com o uso de dexmedetomidina como adjuvante em anestesia perineural em animais e em humanos, constataram maior duração sensitiva, motora e analgésica em bloqueios auxiliados com essa medicação (Brummet et al., 
2009; Brummet et al., 2010; Esmaoglu et al., 2010; Marhofer et al., 2013). Fanelli et al., (1998), observaram que a ropivacaína $0,75 \%$ produziu início de ação mais rápido do que bupivacaína 0,5\%. Duma et al. (2005), relatam não ter diferença significativa ao início de ação sensorial e motora em bloqueios de clonidina associada à bupivacaína, quando comparadas ao grupo controle.

Um estudo realizado por Boscan \& Wennogle (2016), comparou o uso de bloqueio de nervos ciático e femoral com anestesia peridural e sem anestesia regional. Os resultados foram superiores nos grupos submetidos ao bloqueio periférico dos nervos ciático e femoral no que diz respeito ao resgate de analgesia no trans e pós-operatório, bem como, nestes pacientes, foi observado uma recuperação anestésica mais tranquila que os demais grupos. Em contrapartida, o estudo desenvolvido por Bartel et al. (2016), foi realizada a comparação entre a qualidade da anestesia e analgesia pós-operatória de cães submetidos a artroplastia de joelho unilateral, e não foi observada diferença significativa entre os pacientes submetidos a bloqueio de nervo ciático e femoral ou naqueles submetidos a anestesia epidural. Corroborando, McCally et al. (2015), testaram a comparação do uso de bupivacaína epidural e com bloqueio de nervo ciático e femoral, em cães submetidos a osteotomia de nivelamento platô tibial, não encontraram diferença significativa na analgesia pós-operatória imediata.

A ruptura de ligamento cruzado (RLC) é uma afecção bastante frequente na clínica cirúrgica de pequenos animais. A TPLO é um dos procedimentos cirúrgicos comumente realizados, com o intuito de estabilizar o joelho após a ruptura ligamentar. Este procedimento culmina em inúmeros estímulos dolorosos, sendo que as complicações pós-operatórias são correlacionadas com a fisiopatologia dolorosa, o que pode estimular ao desenvolvimento da dor crônica (Katz \& Seltzer, 2009).

Em um estudo realizado por Cardoso (2015), no qual foi feita a comparação entre o bloqueio perineural do nervo ciático e femoral com lidocaína $2 \%$ com bloqueio peridural com o mesmo fármaco, em cães submetidos a correção cirúrgica de RLC, foi demonstrado que, aqueles pacientes que receberam bloqueio perineural necessitaram de maior resgate analgésico em diversos momentos ao longo do procedimento, diferindo do uso da lidocaína peridural. Os autores sugerem duas hipóteses; a primeira, diz respeito a falha na execução da técnica de bloqueio ciático e femoral, independentemente da localização dos nervos por eletroestimulação, causando analgesia ausente ou incompleta e a outra de que esta mesma técnica não produz insensibilização completa das estruturas anatômicas que compõem a articulação do joelho. Para Sakura et al. (2010), a ineficácia do bloqueio destes dois nervos em cirurgia de correção de ruptura de ligamento cruzado em humanos, é relatada devido a necessidade de bloqueio do nervo obturador para que haja uma completa analgesia da articulação femorotibiopatelar.

Um estudo realizado por Cathasaigh et al. (2018), que utilizaram bupivacaína para bloqueio de nervo femoral e ciático, induziu níveis de bloqueio sensorial e motor clinicamente relevantes no membro pélvico afetado por até 20 horas após a administração. Levando em consideração a duração dos procedimentos cirúrgicos ortopédicos do membro pélvico, espera-se que o bloqueio dos nervos femoral e ciático sejam eficazes na anestesia balanceada, bem como na analgesia pós-operatória, reduzindo assim a utilização de analgésicos no pósoperatório (Campoy et al., 2012b; Caniglia et al., 2012).

Campoy et al. (2012b), observaram que cães que receberam o bloqueio de nervos femoral e ciático, quando associada a anestesia geral com propofol e dexmedetomidina, diminuiu o requerimento de fármacos analgésicos adicionais no trans-operatório. A analgesia promovida pelo bloqueio loco-regional perdurou, pelas próximas 10 horas após o bloqueio, chegando a 24 horas em seis cães, os quais não necessitaram de analgesia adicional.

Campoy et al. (2012a), notaram que o intervalo entre o primeiro resgate analgésico, quando os animais foram submetidos a cirurgia de osteotomia tibial, fazendo uso da técnica de bloqueio perineural do nervo ciático e femoral, foi menor do que aqueles submetidos a anestesia peridural com bupivacaína e morfina. Já Caniglia et al. (2012), não notaram diferenças nesta mesma variável, quando administraram lidocaína $1 \%$ com bupivacaína em bloqueios perineurais de ciático e femoral comparado com epidural.

\section{Intercorrências}

Como qualquer outro método anestésico, a anestesia loco-regional também possui algumas 
complicações, sendo que estas estão associadas a concentrações plasmáticas elevadas do anestésico local, grau de absorção, local da aplicação, uso concomitante com adrenalina e viés do animal (Valverde, 2008). Raramente efeitos adversos podem ocorrer, como irritação tecidual, reações alérgicas, bem como reações sistêmicas (Tranquilli \& Skarda, 2007).

Segundo Campoy (2006), as complicações são raras, principalmente quando se faz uso de ENP e US. A complicação frequentemente observada é devido a escolha inadequada da solução anestésica (volume e concentrações inferiores), escolha incorreta dos nervos a serem bloqueados ou técnica incorreta. O acesso difícil aos pontos de referência é fator considerável para falha. Hematomas por punção vascular acidental, punções ou infusões interneurais são complicações mais severas.

Vettorato et al. (2013), relatam a ocorrência de paralisia contralateral, e justificam por ser possível que a solução anestésica tenha migrado para o espaço intervertebral em L7, alcançando assim, a cauda equina e anestesiando o membro pélvico não tratado.

Gurney \& Leece (2014) referem que, durante o procedimento cirúrgico podem ser encontradas alterações cardiorrespiratórias, caso um dos bloqueios seja falho, porém identificar qual dos bloqueios é o responsável é trabalhoso, pois a técnica operatória aplicada pode afetar diversas vias sensoriais do joelho concomitantemente. A fim de testar a falha na técnica anestésica, os pacientes devem ser examinados após a recuperação com o uso de dermátomos.

\section{Conclusão}

Prorrogar o tempo de analgesia em bloqueios perineurais dos nervos femoral e ciático, pode reduzir a necessidade de analgésicos sistêmicos no pós-operatório, reduzindo também as chances de efeitos colaterais observados quando se usa anestesia geral ou epidural. Com o intuito de evitar que erros possam ocorrer durante o procedimento do bloqueio, deve-se usar ultrassom ou neuroestimuladores como auxiliares na localização nervosa. Porém, mesmo com a assistência destes, intercorrências podem ser observadas, porém são raras. Muitos autores comprovaram a eficácia do uso do bloqueio locoregional dos nervos femoral e ciático, como adjuvantes no limiar da dor pós-operatória de pacientes submetidos a procedimentos cirúrgicos ortopédicos, considerados promotores de dor moderada a severa. Porém, para saber se os bloqueios dos nervos femoral e ciático são superiores à anestesia epidural, na redução da dor, maiores estudos precisam ser realizados.

\section{Referências}

Anil, S. S., Anil, L. \& Deen, J. 2002. Challenges of pain assessment in domestic animals. Journal of the American Veterinary Medical Association, 220(3), 313-319.

Bartel, A. K., Campoy, L., Martin-Flores, M, Gleed, R. D., Walker, K. J., Scanapico, C. E. \& Reichard, A. B. 2016. Comparison of bupivacaine and dexmedetomidine femoral and sciatic nerve bloks with bupivacaine and buprenorphine epidural injection for stifle arthrosplasty in dogs. Veterinary Anaesthesia and Analgesia, 43(4), 435-443.

Becker, D. E. \& Reed, K. L. 2012. Local anesthetics: review of pharmacological considerations. Anesthesia Progress, 59(2), 90-101.

Boscan, P. \& Wennogle, S. 2016. Evaluation femoral-sciatic nerve bloks, epidural analgesia, and no use of regional analgesia in dogs undergoing tíbia-plateau-leveling-osteotomy. Journal of the Americon Animal Hospital Association, 52(2), 102-108.

Brummet, C., Padda, A. K., Amodeo, F. S., Welch, K. B. \& Lydic, R. 2009. Perineural dexmedetomidine added to ropivacaine causes a dose-dependent increase in the duration of thermal antinociception in sciatic nerve block in rat. Anesthesiology, 111(15), 1111-1119.

Brummet, C. M., Amodeo, F. S., Janda, A. M., Padda, A. K. \& Lydic, R. 2010 Perineural dexmedetomidine provides an increased duration of analgesia to a thermal stimulus when compared with a systemic control in a rat sciatic nerve block. Regional Anesthesiology Pain Medicine, 35, 427-431.

Bufalari, A., Adami, C., Angeli, G. \& Short, C.E. 2007. Pain assessment in animals. Veterinary Research Communications, 31, 55-58.

Campoy, L, Martin-Flores, Ludders, J. W. \& Gleed, R. D. 2012a. Comparison of bupivacaine femoral and sciatic nerve block versus bupivacaine and morphine epidural for stifle surgery in dogs. Veterinary Anaesthesia and Analgesia, 39(1), 91-98.

Campoy, L, Martin-Flores, Ludders, J. W. \& Gleed, R. D. 2012b. Procedural sedation combined with locoregional anesthesia for 
orthopedic surgery of the pelvic limb in 10 dogs: case series. Veterinary Anaesthesia and Analgesia, 39(4), 436-440.

Campoy, L., Bezuidenhout, A. J., Gleed, R. D., Martin-Flores, M., Raw, R.M., Santare, C.L., Jay, A. R. \& Wang, A. L. 2010. Ultrasoundguided approach for axillary brachial plexus, femoral nerve, and sciatic nerve blocks in dogs. Veterinary Anaesthesia and Analgesia, 37(2), 144-153.

Campoy, L., Martin-Flores, M., Looney, A. L., Erb, H. N., Ludders, J. W., Stewart, J. E., Gleed, R. D. \& Asakawa, M. 2008. Destribuition of lidocaine-methylene blue solution staining in brachial plexus, lumbar plexus and sciatic nerve bloks in the dog. Veterinary Anaesthesia and Analgesia, 35(4), 348-354.

Campoy, L. 2006 Fundamentals of regional anesthesia using nerve stimulation in the dog. In: Gleed, R. D. \& Ludders, J. W. Recent advances in veterinary anesthesia and analgesia: companion animals. International Veterinary Information Service, Ithaca, New York, US.

Caniglia, A. M. Driessen, B., Puerto, D. A., Bretz, B., Boston, R. C. \& Larenza, M. P. 2012. Intraoperative antinociception and postoperative analgesia following epidural anesthesia versus femoral and sciatic nerve blockade in dogs undergoing stifle joint surgery. Journal of the American Veterinary Medical Association, 241(12), 1605-12.

Cardoso, G. S. 2015. Avaliação da anestesia peridural e do bloqueio perineural dos nervos ciático e femoral com lidocaína $2 \%$ em cães anestesiados pelo isofluorano e submetidos à cirurgia de correção de ruptura de ligamento cruzado cranial. Universidade Estadual Paulista "Júlio de Mesquita Filho", Câmpus de Botucatu, 84f

Cathasaigh, M. O, Read, M. R., Atilla, A., Schiller, T. \& Kwong, G. P. S. 2018. Blood concentration of bupivacaine and duration of sensory and motor block following ultrasoundguided femoral an sciatic nerve blocks in dogs. Plos One, 13(3), 1-14.

Chery, J., Semaan, E., Darji, S., Briggs, W. T., Yarmush, J. \& D'Ayala, M. 2014. Impact of regional versus general anesthesia on the clinical outcomes of patients undergoing major lower extremity amputation. Annals of Vascular Surgery, 28(5), 1149-1156.
Conrick-Martin, I. \& Buggy, D. J. 2013. The effects of anesthetic and analgesic techniques on immune function. Journal of Clinical Anesthesia, 25(4), 253- 254.

Costa-Farré, C, Blanch, X. L., Cruz, J. L. \& Franch, J. 2011. Ultrasound guidance for the performance of sciatic and saphenous nerve blocks in dogs. Veterinary Journal, 187(2), 221-224.

Davis, N., Lee, M., Lin, A.Y., Lynch, L., Monteleone, M., Falzon, L., Ispahany, N. \& Lei, S. 2014. Postoperative cognitive function following general versus regional anesthesia: a systematic review. Journal of Neurosurgical Anesthesiology, 26(4), 369-376.

Duma, A., Urbanek, B., Sitzwohl, C., Kreiger, A., Zimpfer, M. \& Kapral, S. 2005. Clonidine as an ajuvant to local anaesthetic axillary brachial plexus block: a rondomized, controlled study. British Journal of Anaesthesia, 94(1), 112-116.

Esmaoglu, A., Yegenoglu, F., Akin, A. \& Turk, C.Y. 2010. Dexmedetomidine added to levobupivacaine prolongs axillary brachial plexus block. Anaesthesia and Analgesia, 111(6), 1548-1551.

Evans, H. E. \& De Lahunta, A. 2010. Vessels and nerves of the pelvic limb. In: Guide to the dissection of the dog (p. 232-255). Saunders, Ithaca, New York, US.

Ferreira, F. M. \& Obertz, L. 2013. Fisiologia do sistema nervoso periférico. In: Klaumann, P. R. \& Otero, P. E. Anestesia locorregional em pequenos animais (cap. 1, p. 1-22). Roca, São Paulo, Brasil.

Fanelli, G., Casati, A., Beccaria, P., Aldegheri, B., Berti, M., Tarantino, F. \& Torri, G. 1998. A Double-Blind Comparison of Ropivacaine, Bupivacaine, and Mepivacaine During Sciatic and Femoral Nerve Blockade. Anesthesia \& Analgesia. 87(3), 597-600.

Flôr, P. B., Martins, T. L. \& Yazbek, K. V. B. 2011. Avaliação da dor. In: Fantoni, D. T. Tratado da dor na clínica de pequenos animais. Elsevier, Rio de Janeiro, BR.

Gaynor, J. S. 1999 Is postoperative pain management important in dogs and cats? Veterinary Medicine, 3(4), 254-257.

Graff, S. M., Wilson, D. V. Guiot, L. P. \& Nelson, N. C. 2015. Comparison of three ultrasound guided approaches to the lumbar plexus in dogs: a cadaveric study. Veterinary Anaesthesia and Analgesia, 42(4), 394-404. 
Gurney, M. A. \& Leece, E. A. 2014. Analgesia for pelvic limb surgery. A review of perpheral nerve bloks and the extradural tchnique. Veterinary Anaesthesia and Analgesia, 41(5), 445-458.

Hoelzler, M. G., Harney, R. C., Lidbetter, D. A. \& Millis, D. L. 2005. Comparison of perioperative analgesic protocols for dogs undergoing tibial plateau leveling osteotomy. Veterinary Surgery, 34(4) 337-44.

Johnson, A. L. 2013. Fundamentals of Orthopedic Surgery and Fracture Management. In: Fossum, T. W. Small Animal Surgery. Elsevier, Philadelphia, US.

Kahvegian, M. P. 2010. Cirurgia ocular. Técnicas de Anestesia local. In: Fantoni \& Cortopassi (2a ed.). Anestesia em cães e gatos. Rocca, São Paulo, Brasil.

Kurosawa, S. \& Kato, M. 2008. Anesthetics, immune cells, and immune responses. Journal of Anesthesia, 22(3), 263-277.

Mahler, S.P. \& Adogwa, A. 2008. Anatomical and experimental studies of brachial plexus, sciatic, and femoral nerve-location using peripheral nerve stimulation in the dog. Veterinary Anaesthesia and Analgesia, 35(1), 80-89.

Marhoferm, D., Marhofer, D., Kettner, S.C., Marhofer, P., Pils, S., Weber, M. \& Zeitlinger, M. 2013. Dexmedetomidine as an adjuvante to ropivacaine prolongs peripheral nerve block: a volunteer study. British Journal of Anesthesia, 110(3), 438-442.

Marhofer, P. \& Chan, V. W. 2007. Ultrassoundguided regional anestesia: Current concepts and future trends. Anaesthesia \& Analgesia, 104(5), 1265-1269.

McCally, R. E., Bukoski, A., Branson, K. R., Fox, D. B. \& Cook, J. L. 2015. Comparison of shortterm postoperative analgesia by epidural, femoral nerve bloks, or combination femoral and sciatic nerve block in dogs undergoing tibial plateau leveling osteotomy. Veterinary Surgery, 44(8), 983-987.

Mich, P. M. \& Hellyer, P. W. 2009. Métodos objetivos e categóricos para avaliar a dor e analgesia. Manual de controle da dor em medicina veterinária (p. 78-109). MedVet, São Paulo, BR..

Mogicato, G., Layssol-Lamour, C., Mahler, S., Charrouin, M., Boyer, G., Verwaerde, P. \& Jourdan, G. 2015. Anatomical and ultrasonographic study of the femoral nerve within the iliopsoas muscle in beagle dogs and cats. Veterinary Anaesthesia and Analgesia, 42(4), 425-432.

Muir, W. W. 2009. Fisiologia e fisiopatologia da dor. In: Gaynor, J.S. \& Muir III, W.W. Manual de controle da dor em medicina veterinária $(2 \mathrm{a}$ ed., p. 13-41). MedVet, São Paulo, BR.

Nielsen, K. C., Nielsen, K. C., Guller, U., Steele, S. M, Klein, S.M., Greengrass, R. A. \& Pietrobon, R. 2005. Influence of obesity on surgical regional anesthesia in the ambulatory setting: an analysis of 9.038 blocks. Anesthesiology, 102(1), 181-187.

Otero, P. E., Portela, D. A. \& Tarragona, L. 2011. Analgesia transoperatória. In: Fantoni, D.T. Tratamento na clínica de pequenos animais. Elsevier, Rio de Janeiro, Brasil.

Portela, D. A. 2013. Anestesia locorregional do membro pélvico. In: Klaumann, P. R. \& Otero, P. E. Anestesia locorregional em pequenos animais. Roca, São Paulo, Brasil.

Portela, D. A., Otero, P.E., Tarragona, L., Briganti, A., Breghi, G. \& Melanie, P. 2010. Combined paravertebral plexus blok and parasacral sciatic block in healthy dogs. Veterinary Anaesthesia and Analgesia, 37(6), 531-541.

Portela, D. A., Otero, P. E., Briganti, A., Romano, M., Corletto, F. \& Breghi, G. 2013. Femoral nerve blok: a novel psoas compartment lateral pre-iliac approach in dogs. Veterinary Anaesthesia and Analgesia, 40(2), 194-204.

Rodriguez, J., Bárcena, M., Taboada-Muñiz, M., Lagunilla, J. \& Alvarez, J. 2004. A comparison of single versus multipe injections on the extent of anestesia with coracoid infraclavicular brachial plexus block. Anaesthesia \& Analgesia, 99(4), 1225-1230.

Sakura, S., Hara, K., Ota, J. \& Tadenuma, S. 2010. Ultrasound-guided peripheral nerve blocks for anterior cruciate ligament reconstruction: effect of obturator nerve block during and after surgery. Journal of Anesthesia, 24(3), 411-7.

Schroeder, K. 2013. History of Regional Anesthesia. In: Campoy, L. \& Read, M. R. Small Animal Regional Anesthesia and Analgesia, Wiley-Blackwell Publishing, Oxford, UK.

Silva, A., Guedes, A. \& Assunção, J. P. 2011. Avaliação Neurológica em Anestesia LocoRegional. Revista de Anestesia Regional e Terapia da Dor, 64, 11-15 
Tayari, H., Tazioli, G. Breghi, G. \& Briganti, A. 2017. Ultrasound-guided femoral and obturator nerves block in the psoas compartment in dogs: anatomical and randomized clinical study. Veterinary Anaesthesia and Analgesia, 44(5), 1216-1226.

Tranquilli, W. J. \& Skarda, R. T. 2007. Local anesthetics. Veterinary Anaesthesia and Analgesia. Blackwell Publishing, Iowa, US.

Treede, R. D., Jensen, T. S., Campbell, J. N., Cruccu, G., Dostrovsky, J. O., Griffin, J. W. ... \& Serra, J. 2008. Neuropatic pain: redefinition and a granding system for clinical and research purposes. Neurology, 70(18), 1630-1635.

Valverde, A. 2008. Epidural analgesia and anesthesia in dogs and cats. Veterinary Clinics of North America: Small Animal Practice, 38(6), 1205-1230.

Vettorato, E., De Gennaro, C. Okushima, S \& Corletto, F. 2013. Retrospective comparison of two peripheral lumbosacral plexus blocks in dogs undergoing pelvic limb orthopedic surgery. Journal of Small Animal Practice British Small Animal Veterinary Association, 54(12), 630-637.

Vettorato, E., Bradbrook, C., Gurney, M., Aprea, F., Clark, L. \& Corletto, E. 2012. Peripheral nerve blocks of the pelvic limb in dogs: a retrospective clinical study. Veterinary and Comparative Orthopedics Traumatology, 25(4), 314-320.

Xu, J. \& Brennan, T. J. 2011. The pathophysiology of acute pain: animal models. Current Opinion in Anaesthesioly, 24(5) 508514.

Recebido: 15 Julho. 2018

Aprovado: 13 Agosto. 2018

Publicado: 12 Setembro. 2018

Licenciamento: Este artigo é publicado na modalidade Acesso Aberto sob a licença Creative Commons Atribuição 4.0 (CC-BY 4.0), a qual permite uso irrestrito, distribuição, reprodução em qualquer meio, desde que o autor e a fonte sejam devidamente creditados. 